\title{
BMJ Open Safety and efficacy of tranexamic acid in bleeding paediatric trauma patients: a systematic review protocol
}

Denisa Urban, ${ }^{1}$ Ruben Dehaeck, ${ }^{2}$ Diane Lorenzetti, ${ }^{3,4,5}$ Jonathan Guilfoyle, ${ }^{6}$ Man-Chiu Poon, ${ }^{7,8,9}$ MacGregor Steele, ${ }^{10}$ David Lardner, ${ }^{11}$ Irene Wai Yan Ma, ${ }^{12}$ Mary Elizabeth Brindle ${ }^{2,4}$

To cite: Urban D, Dehaeck R, Lorenzetti $\mathrm{D}$, et al. Safety and efficacy of tranexamic acid in bleeding paediatric trauma patients: a systematic review protocol. BMJ Open 2016;6: e012947. doi:10.1136/ bmjopen-2016-012947

- Prepublication history and additional material is available. To view please visit the journal (http://dx.doi.org/ 10.1136/bmjopen-2016012947).

Received 6 June 2016 Revised 25 August 2016 Accepted 2 September 2016

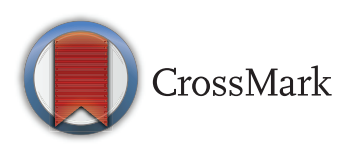

For numbered affiliations see end of article.

Correspondence to Mary Elizabeth Brindle; maryebrindle@gmail.com

\section{ABSTRACT}

Introduction: Trauma is the leading cause of death among children aged 1-18. Studies indicate that better control of bleeding could potentially prevent $10-20 \%$ of trauma-related deaths. The antifibrinolytic agent tranexamic acid (TXA) has shown promise in haemorrhage control in adult trauma patients. However, information on the potential benefits of TXA in children remains sparse. This review proposes to evaluate the current uses, benefits and adverse effects of TxA in the bleeding paediatric trauma population. Methods and analysis: A structured search of bibliographic databases (eg, MEDLINE, EMBASE, PubMed, CINAHL, Cochrane CENTRAL) has been undertaken to retrieve randomised controlled trials and cohort studies that describe the use of TxA in paediatric trauma patients. To ensure that all relevant data were captured, the search did not contain any restrictions on language or publication time. After deduplication, citations will be screened independently by 2 authors, and selected for inclusion based on prespecified criteria. Data extraction and risk of bias assessment will be performed independently and in duplicate. Meta-analytic methods will be employed wherever appropriate.

Ethics and dissemination: This study will not involve primary data collection, and formal ethical approval will therefore not be required. The findings of this study will be disseminated through a peerreviewed publication and at relevant conference meetings.

Trial registration number: CRD42016038023.

\section{INTRODUCTION}

Trauma is a substantial cause of morbidity and mortality in developed and developing countries. $^{1-3}$ In 2013, 973 million people worldwide sustained injuries that required medical attention and 4.8 million of these died from their injuries. ${ }^{4}$ Trauma represents the leading cause of death and potential years of life lost among children and youth under the age of 18 years. ${ }^{5}$ In 2004 , an estimated 950000 children under the age of 18

\section{Strengths and limitations of this study}

- This study is the first systematic review to evaluate the uses and efficacy of tranexamic acid (TXA) in paediatric trauma patients.

- This study has a great potential for translation as current protocols in paediatric trauma are extrapolated from adult studies or generated from small diverse paediatric studies.

- The aims and objectives of this study were set by relevant stakeholders, including a paediatric surgeon, a paediatric anaesthesiologist, a paediatric emergency physician and two haematologists.

- We anticipate that there will be few trials specifically evaluating the use of TXA in paediatric trauma patients and the numbers of patients within studies are likely to be small. The data available may be heterogenous and not suitable for meta-analysis.

died of an injury worldwide. ${ }^{6}$ In the USA in 2012, 6.8 million children 18 years of age and younger were treated for trauma-related disorders, resulting in a total medical expenditure of US $\$ 7.8$ billion. ${ }^{7}$

Haemorrhage continues to be a significant contributor to trauma-related death. ${ }^{8}$ Studies indicate that better control of bleeding could prevent $10-20 \%$ of these deaths. ${ }^{9}$ Vascular injury normally triggers coagulation, a complex proteolytic cascade that culminates in the formation of fibrin, a critical factor in control of bleeding. ${ }^{10}$ In the presence of tissue trauma and systemic hypoperfusion, the process is disrupted, leading to the development of acute traumatic coagulopathy (ATC). ${ }^{11}$ Continued blood loss, acidaemia, hypothermia, consumption of clotting factors and haemodilution resulting from resuscitation efforts can exacerbate the coagulation defect. $^{12}$ Approximately $25 \%$ of severely injured patients present with ATC, ${ }^{13} 14$ a condition associated with higher transfusion requirements, organ failure, septic complications, 
increased length of stay in the intensive care unit (ICU) and mortality. ${ }^{15}{ }^{16}$ Consequently, management of ATC has become a prominent issue in the care of trauma patients. ${ }^{17}$ Tranexamic acid (TxA), an antifibrinolytic agent, has shown promise in haemorrhage control in adult trauma patients. As a synthetic derivative of the amino acid lysine, TxA competitively inhibits the activation of plasminogen to plasmin, a serine protease that breaks down fibrin and prevents blood clot formation. ${ }^{18}$ In the large randomised CRASH-2 trial with over 20000 participants 16 years of age and older, the use of TxA was associated with a significant reduction in mortality rates due to bleeding, and was demonstrated to be especially beneficial when administered within 1-hour of injury. ${ }^{19} 20$ Survival benefits and reduced coagulopathy were also observed in the military setting, where the MATTERS I and II cohort studies of more than 2000 combat casualties used TxA in emergency resuscitation. $^{2122}$ Neither study identified significant risks, such as increased thromboembolic events, associated with the administration of TxA.

Multiple studies in paediatric patients have demonstrated the benefits of TxA in major spine, cardiac and craniofacial surgeries. Meta-analyses on the uses of TxA in paediatric surgery concluded that the antifibrinolytic led to decreased blood loss and a reduced need for blood product transfusion. ${ }^{23-26}$ However, the pathogenesis of traumatic haemorrhage differs significantly from non-traumatic surgical bleeding. During the former, tissue injuries are massive, uncontrolled and patients commonly experience delays before receiving treatment. ${ }^{25}$ While elective surgeries are performed in a controlled environment, where normothermia and normovolaemia are maintained, ${ }^{27} 28$ hypothermia and hypovolaemic shock are observed frequently in the traumatically injured patient. ${ }^{29}$ It is therefore unlikely that the methods employed to control surgical bleeding will be directly applicable to the control of traumatic haemorrhage, and a knowledge synthesis that explores the specific role of TxA in paediatric trauma is warranted.

Recently, the PED-TRAX observational study of 766 injured patients 18 years of age or younger showed decreased mortality, improvements in discharge neurological status as well as decreased ventilator dependence in bleeding trauma patients treated with TxA compared with those who were not. Furthermore, no adverse safety-related or medication-related complications were observed. $^{30}$ In recognition of the potential life-saving effects of TxA, the Royal College of Paediatrics and Child Health has issued a recommendation on a pragmatic dosage schedule for injured children. ${ }^{31}$ The American Academy of Pediatrics, however, has not yet issued any official guidelines on the use of TxA in paediatric traumatic injury. While some children's hospitals have integrated TxA into their mass transfusion protocols, a consensus on its timing and dosing has not yet been reached. ${ }^{8}{ }^{32}$

This review proposes to address the observed gap in current paediatric trauma care by investigating the uses, benefits and adverse effects of TxA in injured children. The synthesis will collate existing evidence on the efficacy of TxA and facilitate the development of novel trauma care pathways and policies directed at the injured child. There is likely to be a small number of studies that meet inclusion criteria, but the PED-TRAX study represents at least one large cohort study that should be included within the synthesis. ${ }^{30}$

\section{Systematic review question}

- What is the current evidence of effectiveness of TxA at reducing morbidity and mortality in traumatically injured children with haemorrhage?

- What are the current uses, benefits, dosing regimens and adverse effects of TxA compared with standard practice for controlling traumatic haemorrhage in paediatric patients?

\section{METHODS AND ANALYSIS}

This review will follow the guidelines and recommendations laid out by the Cochrane Collaboration ${ }^{33}$ and will conform to the standards of the Preferred Reporting Items for Systematic Reviews and Meta-analyses (PRISMA). The protocol was developed according to the Preferred Reporting Items for Systematic Reviews and Meta-analyses Protocols (PRISMA-P) (see online supplementary table S1). The review has been registered with PROSPERO under the identification code: CRD42016038023.

\section{Eligibility criteria}

Population

To be included, studies must describe participants younger than 18 years of age who have suffered any type of traumatic injury with haemorrhage. Studies of patients with pre-existing haematological disorders will be excluded.

\section{Intervention}

Studies that describe the administration of any amount of TxA, whether intravenously as a bolus and/or infusion, to control haemorrhage in trauma patients younger than 18 years of age within the first 24 hours after injury will be included. Studies that describe coadministration of other antifibrinolytic agents with TxA will be excluded, as this may confound results. Studies that describe the use of TxA in elective surgeries will be excluded.

\section{Comparator}

For inclusion, studies must contain a comparator group of paediatric trauma patients that have received routine measures to control haemorrhage or who have also received a placebo. Studies that compare different TxA dosing schemes and no placebo, or studies that compare the efficacy of TxA with other antifibrinolytics (eg, $\varepsilon$-aminocaproic acid, recombinant factor VIIa, aprotinin), will also be considered. 


\section{Setting}

Studies will not be restricted to location or setting. All circumstances where TxA was administered to bleeding trauma patients will be examined.

\section{Types of study to be included}

To be included, studies must be randomised controlled trials or clinical trials. Cohort studies will be considered for inclusion, provided that data from a comparison group are reported. Case or series studies will be excluded due to the risk of bias associated with these study designs. Case-control studies, with the exceptions of those nested as part of a cohort study, will be excluded. Economic evaluation studies will be excluded.

\section{OUTCOMES}

The primary outcome of interest will be death within 4 weeks of injury. Secondary outcomes will include the number of units of blood products transfused, rebleeding events, surgical intervention and the occurrence of thromboembolic events. The method, dosing and timing of TxA administration will also be examined within subgroup analyses.

\section{Search strategy}

A search strategy was created with the aid of a research librarian and combines keywords and MeSH terms from three themes: the population (paediatric patients), the condition (trauma and haemorrhage) and the intervention (TxA) (see online supplementary table S2). Search terms were created as keywords (title/abstract words) and subject headings (eg, MeSH) as appropriate. No language or publication date restrictions will be placed on the searches. The searches will be kept broad, and the search strategies created have incorporated neither study design filters nor limitations on comparators or outcomes. Sources to be searched will include bibliographic databases (MEDLINE, EMBASE, PubMed, CINAHL, Cochrane CENTRAL); abstract and conference proceedings resources (Web of Science Conference Proceedings Citation Index-Science (1990-present), Canadian Anesthesiologists' Society, Trauma Care Conference, The Canadian Association of Emergency Physicians) and controlled trials registries (clinicaltrials. gov, WHO International Clinical Trials Registry Platform (ICTRP), ISRCTN registry, HKU Clinical Trials Registry, Clinical Trials Registry-India, UK Clinical Trials Gateway, Canadian Clinical Trials Database). Additional citations will be retrieved by scanning the reference lists of included studies and relevant reviews, and contacting authors of key publications and other experts in this field.

\section{Study selection and quality assessment}

Citations will be collected in a reference manager software program (EndNote) and duplicates will be eliminated by first using the CREBP Systematic Review
Assistant tool (crebp-sra.com) and then manually. The full list of citations will be screened by two reviewers independently and in duplicate in two stages, the first restricted to citation titles and abstracts, followed by fulltext review of included articles. Inter-rater agreement will be assessed through Cohen's $\kappa$ coefficient. ${ }^{34}$ For this statistic, values $<0.4$ indicate poor agreement, $0.4-0.59$ fair agreement, $0.60-0.74$ good agreement and $>0.75$ excellent agreement. ${ }^{35}$ Discrepancies between the reviewers will be resolved by discussion or with the assistance of a third team member. The risk of bias assessment will be conducted using the recommendations and tools provided by the Cochrane Collaboration. ${ }^{33}$

\section{Data extraction and analysis}

Basic information about included studies will be collected (authors, journal, years of study and publication, location of study, study design, number of patients included, etc). Patient demographics will be recorded along with type of injury and severity (eg, injury severity score, Glasgow Coma Scale, etc), and haemostatic measurements (eg, prothrombin time, haemoglobin levels, clot lysis at $30 \mathrm{~min}$, etc). The method, timing and dosing for TxA administration will be recorded. Outcome data to be abstracted will include mortality at 24 and 48 hours, at 4 weeks postinjury and at/before discharge, cause of death, blood product administration, incidence of thromboembolic events, rebleeding and surgical intervention.

Data abstraction will be conducted by two reviewers independently and in duplicate with the aid of a standardised, piloted data extraction form. Disagreements between the two reviewers will be resolved through discussion or with the assistance of a third team member.

Data analysis will take the form of a narrative synthesis in which studies are described and categorised according to design and purpose. Subgroup analysis may involve stratification of studies by types of injury or TxA administration (bolus vs continuous intravenous infusion) and dosage. A meta-analysis will be conducted if effect measures for individual studies are provided or can be calculated and if studies are suitably homogeneous. The degree of heterogeneity will be determined through a qualitative assessment of the clinical diversity and methodological heterogeneity. The degree of heterogeneity will be evaluated qualitatively as well as quantitatively through the use of the $\mathrm{I}^{2}$ statistic: $\mathrm{I}^{2}=(\mathrm{Q}-\mathrm{df} / \mathrm{Q}) \times 100 \%$.

Meta-analysis using a random-effects model will be considered in the case of moderate or unimportant heterogeneity $\left(\mathrm{I}^{2} \leq 60 \%\right)$. In the case of substantial heterogeneity, the analysis will be restricted to the narrative synthesis exploring heterogeneity through subgroup analysis.

\section{Confidence in cumulative evidence and assessment of publication bias}

The GRADE system will be employed to assess the quality of the evidence presented across the studies included for review. ${ }^{36}$ Depending on design, methods 
and execution, consistency and directness of evidence, quality of evidence from each study will be rated as high, moderate, low or very low by two independent reviewers. Disagreements will be resolved through discussion or with the assistance of a third team member. The GRADE system will also be employed to evaluate any recommendations that will arise from this review.

Publication bias will be assessed through visual inspection of the Begg's funnel plot and through the use of Egger's test.

\section{DISCUSSION}

This knowledge synthesis proposes to collate existing evidence on the current uses, safety and effectiveness of TxA in the control of haemorrhage in injured children. We anticipate that our data synthesis will provide sufficient information to inform trauma care pathways and assist clinicians caring for paediatric trauma patients. Finally, this systematic review will help expand our understanding of the uses, benefits and harms of antifibrinolytic drugs in the bleeding trauma patient and identify areas where gaps in knowledge remain.

\section{Author affiliations}

${ }^{1}$ Cumming School of Medicine, University of Calgary, Calgary, Alberta, Canada ${ }^{2}$ Department of Surgery, Cumming School of Medicine, University of Calgary, Calgary, Alberta, Canada

${ }^{3} 0$ 'Brien Institute for Public Health, University of Calgary, Calgary, Alberta, Canada

${ }^{4}$ Department of Community Health Sciences, Cumming School of Medicine, University of Calgary, Calgary, Alberta, Canada

${ }^{5}$ Institute of Health Economics, Edmonton, Alberta, Canada

${ }^{6}$ Division of Pediatric Emergency Medicine, Department of Pediatrics, Cumming School of Medicine, University of Calgary, Calgary, Alberta, Canada ${ }^{7}$ Division of Hematology/Hematologic Malignancies, Department of Medicine, Cumming School of Medicine, Calgary, Alberta, Canada

${ }^{8}$ Division of Hematology/Hematologic Malignancies, Department of Pediatrics, Cumming School of Medicine, Calgary, Alberta, Canada

${ }^{9}$ Division of Hematology/Hematologic Malignancies, Department of Oncology, Cumming School of Medicine, Calgary, Alberta, Canada

${ }^{10}$ Section of Pediatric Hematology, Department of Pediatrics, Cumming School of Medicine, University of Calgary, Calgary, Alberta, Canada

${ }^{11}$ Division of Pediatric Anesthesia, Department of Anesthesia, Cumming School of Medicine, University of Calgary, Calgary, Alberta, Canada

${ }^{12}$ Division of General Internal Medicine, Department of Medicine, Cumming School of Medicine, University of Calgary, Calgary, Alberta, Canada

Twitter Follow Mary E Brindle at @MaryEBrindle

Contributors DU developed and wrote the review protocol and will write the final manuscript. DL assisted with the design of the search strategy. MEB assisted with formulating the aims and objectives of the study and assisted in study design, including determination of the inclusion and exclusion criteria. IWYM has aided in study design, including planning statistical analysis. RD, JG, M-CP, MS and DL aided in setting the aims and objectives of this study. All authors have aided in review and revision of this manuscript and approve of it in its current form.

Funding Research reported in this publication is supported by the Alberta Health Services Emergency Strategic Clinical Network (ESCN) 2016 Systematic Review Grant and also the Brian and Brenda MacNeill Chair in Pediatric Surgery through the EQuIS quality and safety research platform.

Disclaimer The content is solely the responsibility of the authors and does not necessarily represent the official views of the ESCN Scientific Office, the MacNeill family or Alberta Health Services.
Competing interests None declared.

Ethics approval This study will not involve primary data collection, and formal ethical approval will therefore not be required.

Provenance and peer review Not commissioned; externally peer reviewed.

Data sharing statement Although our protocol is a systematic review, we will make available any unpublished data that was involved in our study (ie, completed screening tools and extractions) if requested.

Open Access This is an Open Access article distributed in accordance with the Creative Commons Attribution Non Commercial (CC BY-NC 4.0) license, which permits others to distribute, remix, adapt, build upon this work noncommercially, and license their derivative works on different terms, provided the original work is properly cited and the use is non-commercial. See: http:// creativecommons.org/licenses/by-nc/4.0/

\section{REFERENCES}

1. Murray CJL, Lopez AD, World Health Organization, et al. The Global burden of disease: a comprehensive assessment of mortality and disability from diseases, injuries, and risk factors in 1990 and projected to 2020. Geneva: World Health Organization, 1996.

2. World Health Organization. The global burden of disease: 2004 update. Geneva: World Health Organization, 2008.

3. World Health Organization. Department of Injuries and Violence Prevention. The injury chart book: a graphical overview of the global burden of injuries. Geneva: World Health Organization, 2002.

4. Haagsma JA, Graetz N, Bolliger I, et al. The global burden of injury: incidence, mortality, disability-adjusted life years and time trends from the Global Burden of Disease study 2013. Inj Prev 2016;22:3-18.

5. Prevention CfDCa. Leading Causes of Death Reports, National and Regional, 1999-2014. 2014. http://webappa.cdc.gov/cgi-bin/broker. exe (accessed 26 Apr 2016)

6. Peden M, World Health Organization. World report on child injury prevention. Geneva: World Health Organization, 2008.

7. Soni A. Top five most costly conditions among children, ages $0-17$, 2012: estimates for the U.S. civilian noninstitutionalized population. 2015. http://meps.ahrq.gov/mepsweb/data_files/publications/st472/ stat472.pdf (accessed 26 Apr 2016).

8. Beno S, Ackery AD, Callum J, et al. Tranexamic acid in pediatric trauma: why not? Crit Care 2014;18:313.

9. Moore FA, McKinley BA, Moore EE. The next generation in shock resuscitation. Lancet 2004;363:1988-96.

10. Schenone M, Furie BC, Furie B. The blood coagulation cascade. Curr Opin Hematol 2004;11:272-7.

11. Brohi K, Cohen MJ, Ganter MT, et al. Acute coagulopathy of trauma: hypoperfusion induces systemic anticoagulation and hyperfibrinolysis. J Trauma 2008;64:1211-17; discussion 17.

12. Davenport R. Pathogenesis of acute traumatic coagulopathy Transfusion 2013;53(Suppl 1):23S-7S.

13. Brohi K, Singh J, Heron M, et al. Acute traumatic coagulopathy. J Trauma 2003;54:1127-30.

14. MacLeod JB, Lynn M, McKenney MG, et al. Early coagulopathy predicts mortality in trauma. J Trauma 2003:55:39-44.

15. Maegele M, Schöchl H, Cohen MJ. An update on the coagulopathy of trauma. Shock 2014;41(Suppl 1):21-5.

16. Cap A, Hunt B. Acute traumatic coagulopathy. Curr Opin Crit Care 2014;20:638-45.

17. Dzik WH, Blajchman MA, Fergusson D, et al. Clinical review: Canadian National Advisory Committee on Blood and Blood Products-massive transfusion consensus conference 2011: report of the panel. Crit Care 2011;15:242.

18. Astedt B. Clinical pharmacology of tranexamic acid. Scand $J$ Gastroenterol Suppl 1987;137:22-5.

19. Williams-Johnson JA, McDonald AH, Strachan GG, et al. Effects of tranexamic acid on death, vascular occlusive events, and blood transfusion in trauma patients with significant haemorrhage (CRASH-2): a randomised, placebo-controlled trial. West Indian Med J 2010;59:612-24.

20. Roberts I, Shakur H, Afolabi A, et al. CRASH-2 Collaborators. The importance of early treatment with tranexamic acid in bleeding trauma patients: an exploratory analysis of the CRASH-2 randomised controlled trial. Lancet 2011;377:1096-101, 101 e1-2. 
21. Morrison JJ, Dubose JJ, Rasmussen TE, et al. Military Application of Tranexamic Acid in Trauma Emergency Resuscitation (MATTERs) study. Arch Surg 2012;147:113-19.

22. Morrison JJ, Ross JD, Dubose JJ, et al. Association of cryoprecipitate and tranexamic acid with improved survival following wartime injury: findings from the MATTERs II study. JAMA Surg 2013;148:218-25.

23. Schouten ES, van de Pol AC, Schouten AN, et al. The effect of aprotinin, tranexamic acid, and aminocaproic acid on blood loss and use of blood products in major pediatric surgery: a meta-analysis. Pediatr Crit Care Med 2009;10:182-90.

24. Grant JA, Howard J, Luntley J, et al. Perioperative blood transfusion requirements in pediatric scoliosis surgery: the efficacy of tranexamic acid. J Pediatr Orthop 2009;29:300-4.

25. Faraoni D, Goobie SM. The efficacy of antifibrinolytic drugs in children undergoing noncardiac surgery: a systematic review of the literature. Anesth Analg 2014;118:628-36.

26. Faraoni D, Willems A, Melot $C$, et al. Efficacy of tranexamic acid in paediatric cardiac surgery: a systematic review and meta-analysis. Eur J Cardiothorac Surg 2012;42:781-6.

27. Sessler DI. New surgical thermal management guidelines. Lancet 2009;374:1049-50.

28. Carless PA, Henry DA, Moxey AJ, et alCell salvage for minimising perioperative allogeneic blood transfusion. Cochrane Database Syst Rev 2006;(4):CD001888
29. Dobson GP, Letson HL, Sharma R, et al. Mechanisms of early trauma-induced coagulopathy: the clot thickens or not? J Trauma Acute Care Surg 2015;79:301-9.

30. Eckert MJ, Wertin TM, Tyner SD, et al. Tranexamic acid administration to pediatric trauma patients in a combat setting: the pediatric trauma and tranexamic acid study (PED-TRAX). J Trauma Acute Care Surg 2014;77:852-8; discussion 58.

31. Royal College of Paediatrics and Child Health. Evidence Statement: major trauma and the use of tranexamic acid in children, November 2012. 2012. https://www.tarn.ac.uk/content/downloads/3100/ 121112_TXA evidence statement_final v2.pdf (accessed 26 Jan 2016).

32. The Alberta Children's Hospital. Massive Transfusion Protocol. 2009. http://iweb.calgaryhealthregion.ca/programs/trauma/pdf/mtp_ oct2009.pdf (accessed 26 Jan 2016).

33. Higgins JP, Green S. Cochrane handbook for systematic reviews of interventions. Wiley Online Library, 2008.

34. McGinn T, Wyer PC, Newman TB, et al. Tips for learners of evidence-based medicine: 3 . Measures of observer variability (kappa statistic). CMAJ 2004;171:1369-73.

35. Cooper H, Hedges LV, Valentine JC. The handbook of research synthesis and meta-analysis. Russell Sage Foundation, 2009.

36. Atkins D, Best D, Briss PA, et al. Grading quality of evidence and strength of recommendations. BMJ 2004;328:1490. 